\title{
Derivation and Identification of Motor Neurons from Human Urine-Derived Induced Pluripotent Stem Cells
}

\author{
Huan Yi, ${ }^{1}$ Bingbing Xie, ${ }^{2}$ Ben Liu, ${ }^{3}$ Xuan Wang, ${ }^{1}$ Li Xu, ${ }^{1}$ Jia Liu, ${ }^{1}$ Min Li, ${ }^{1}$ Xiufeng Zhong (D), ${ }^{2}$ \\ and Fuhua Peng $\mathbb{D}^{1}$ \\ ${ }^{1}$ Department of Neurology, The Third Affiliated Hospital of Sun Yat-sen University, Guangzhou, Guangdong 510630, China \\ ${ }^{2}$ State Key Laboratory of Ophthalmology, Zhongshan Ophthalmic Center, Sun Yat-sen University, Guangzhou, \\ Guangdong 510600, China \\ ${ }^{3}$ Department of Dermatology, The Third Affiliated Hospital of Sun Yat-sen University, Guangzhou, Guangdong 510630, China
}

Correspondence should be addressed to Xiufeng Zhong; zhongxf7@mail.sysu.edu.cn and Fuhua Peng; pfh93@163.com

Received 19 June 2017; Revised 9 September 2017; Accepted 18 September 2017; Published 24 January 2018

Academic Editor: Boon C. Heng

Copyright (C) 2018 Huan Yi et al. This is an open access article distributed under the Creative Commons Attribution License, which permits unrestricted use, distribution, and reproduction in any medium, provided the original work is properly cited.

Induced pluripotent stem cells (iPSCs) have provided new opportunities for motor neuron disease (MND) modeling, drug screening, and cellular therapeutic development. Among the various types of iPSCs, urine-derived iPSCs have become a promising source of stem cells because they can be safely and noninvasively isolated and easily reprogrammed. Here, for the first time, we differentiated urine-derived iPSCs (urine-iPSCs) into motor neurons (MNs) and compared the capacity of urine-iPSCs and cord-blood-derived iPSCs (B-iPSCs) to differentiate into MNs. With the use of small molecules, mature MNs were generated from urine-iPSCs as early as 26 days in culture. Furthermore, in coculture with muscle cells, MNs projected long axons and formed neuromuscular junctions (NMJs). Immunofluorescence and PCR confirmed the expression levels of both MN and NMJ markers. The comparison of the ratios of positive labeling for MN markers between urineiPSCs and B-iPSCs demonstrated that the differentiation potentials of these cells were not significantly different. The abovementioned results indicate that urine-iPSCs are a new, promising source of stem cells for MND modeling and further cellular therapeutic development.

\section{Introduction}

Motor neuron diseases (MNDs) selectively affect motor neurons (MNs), which project axons to muscles and control voluntary actions. Patients with MNDs may present a range of symptoms, such as muscular weakness, atrophy, and hyperreflexia, which ultimately lead to death [1]. No effective treatments are available for MND. Thus, pluripotent stem cells (PSCs) have become an important tool for the study of MND and represent a promising therapeutic approach [2]. Among the variety of stem cells, induced pluripotent stem cells (iPSCs), which are reprogrammed from adult somatic cells, are very advantageous for MND modeling, drug discovery, and individual therapeutic transplantation, without ethical concerns [3]; all of these efforts have made substantial progress toward understand- ing MND. To date, multiple types of somatic cells have been reprogrammed into iPSCs, including widely used skin fibroblasts and peripheral blood cells, which have the potential to differentiate into MNs [4]. Compared with these cells, urinary cells provide a convenient, cost-effective, and noninvasive source of cells that can be obtained and reprogrammed into iPSCs [5]. However, it is unclear whether urine-derived human iPSCs have the capacity to differentiate into MNs. In this study, we rapidly and efficiently induced the differentiation of urine-derived iPSCs (urine-iPSCs) into MNs. Immunofluorescence and PCR confirmed the expression levels of neural markers at every stage of induction and $\mathrm{MN}$-specific markers of cells derived from urine-iPSCs. We also demonstrated the functional capacity of MNs to form NMJs in cocultures of urine-derived MNs and muscle cells. 


\begin{tabular}{|c|c|c|c|c|c|c|c|c|c|}
\hline $\begin{array}{l}\text { Culture } \\
\text { condition }\end{array}$ & Day 0 & & $\begin{array}{c}\text { Day } 7 \\
\square\end{array}$ & & Day 13 & Day 19 & & Day 26 & \\
\hline \multirow[b]{2}{*}{$\begin{array}{l}\text { Small } \\
\text { molecules }\end{array}$} & \multirow{2}{*}{ iPSCs } & Matrigel & \multirow{2}{*}{ NEP } & Matrigel & \multirow{2}{*}{ MNP } & Suspension & \multirow{2}{*}{$\mathrm{MN}$} & Matrigel & \multirow{2}{*}{ Mature MN } \\
\hline & & $\begin{array}{l}\text { CHIR + SB } \\
+ \text { DMH1 }\end{array}$ & & $\begin{array}{l}\text { CHIR + SB } \\
+ \text { DMH1 + RA } \\
+ \text { Pur }\end{array}$ & & $\begin{array}{l}\text { RA + Pur + BDNF } \\
+ \text { GDNF + IGF }\end{array}$ & & $\begin{array}{l}\text { RA + Pur + CpdE } \\
+ \text { BDNF + GDNF } \\
+ \text { IGF }\end{array}$ & \\
\hline
\end{tabular}

(a)

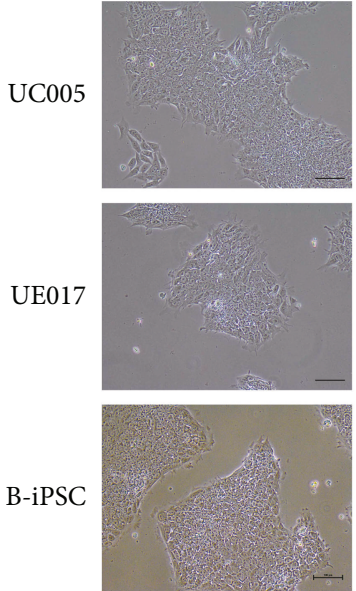

iPSCs
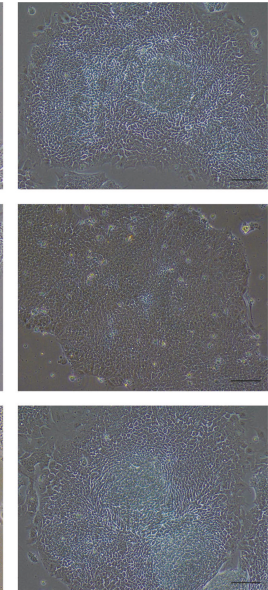

NEP
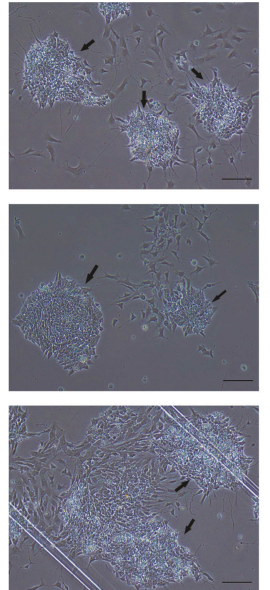

MNP
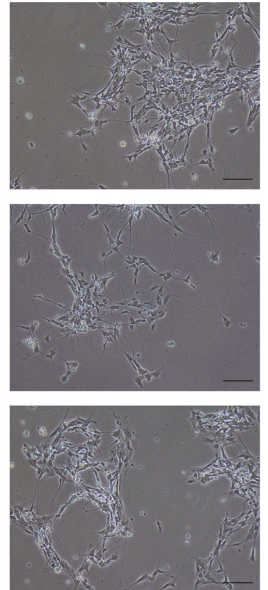

MN
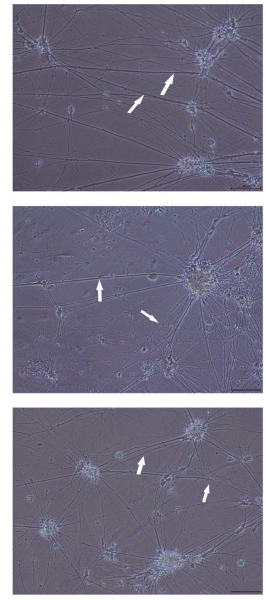

Mature MN

(b)

FIgURE 1: (a) Time course and small-molecule cocktail for the differentiation of iPSCs into mature MNs. Following exposure to CHIR99021, SB431542, and DMH1 for 6 days, the iPSCs differentiated into NEPs. Over 6 days, with the addition of RA and Pur, the cells differentiated into MNPs. During the last 2 weeks of differentiation, in the presence of RA, Pur, and neurotrophic factors, the MNPs finally differentiated into mature MNs. (b) Morphology of the cells derived from the three iPSC lines at every differentiation stage. Before the differentiation process, the three iPSC lines exhibited uniform, undifferentiated morphology. After 6 days of induction, the cells exhibited inconsistent size and shape and aggregated centrally (D7, NEPs). With an additional 6 days of differentiation, cell morphology changed quickly, and the cells started to gather centrally to form rosettes (D13, MNPs, black arrow). On day 19, the differentiated MNs began projecting axons. The MNs matured gradually, and their axons elongated over time (D26, white arrow). The scale bar is $100 \mu \mathrm{m}$.

\section{Materials and Methods}

2.1. iPSC Cultures. The human iPSC lines used in this study included two urine cell-derived iPSC lines, UE017 and UC005, obtained from the Chinese Academy of Sciences, Guangzhou Institute of Biomedicine and Health [6]. As a control, a cord blood-derived iPSC (B-iPSC) line, which was purchased from Gibco (USA, catalog A18945), was used in this study. All iPSCs were cultured on Matrigel-coated plates with mTeSR I (STEMCELL Technologies, Canada), which was changed daily. Immunofluorescence was performed to identify the expression profiles of pluripotency markers.

2.2. Differentiation of iPSCs into MNs. Du's protocol for the differentiation of iPSCs into MNs was used with slight modification (Figure 1(a)) [7]. For MN generation, undifferentiated iPSCs were dissociated with $5 \mu \mathrm{M}$ EDTA (Invitrogen) for $5 \mathrm{~min}$ and then passaged in Matrigel-coated plates in $1: 6$. The following day, the stem cell medium mTeSR was replaced with neural differentiation medium (NDM) with the addition of $3 \mu \mathrm{M}$ CHIR99021 (CHIR, Sigma), $2 \mu \mathrm{M}$ DMH1 (Sigma), and $2 \mu \mathrm{M}$ SB341542 (SB, Sigma). NDM includes Dulbecco's Modified Eagle Medium (DMEM/F12),
Neurobasal Medium at a $1: 1$ concentration, $0.5 \times \mathrm{N} 2$, $0.5 \times$ B27, $0.1 \mathrm{mM}$ ascorbic acid (Sigma), $1 \times$ Glutamax, and $1 \times$ antibiotic-antimycotic (all others from Invitrogen). The medium was changed every other day. IPSCs maintained under these conditions for 6 days differentiated into neuroepithelial progenitors (NEPs). On day 7, the NEPs were treated with dispase $(1 \mathrm{mg} / \mathrm{ml})$ for $5 \mathrm{~min}$ and then gently resuspended with $\mathrm{NDM}$, including $1 \mu \mathrm{M}$ CHIR, $2 \mu \mathrm{M}$ $\mathrm{DMH} 1,2 \mu \mathrm{M}$ SB, $0.1 \mu \mathrm{M}$ retinoic acid (RA, Sigma), and $0.5 \mu \mathrm{M}$ purmorphamine (Pur, Sigma), and were plated on Matrigel-coated plates at a 1:3 concentration. The culture medium was changed every other day for 6 days, and on day 12 , the NEPs differentiated into motor neuron progenitors (MNPs), which aggregated in rosettes. To induce MNPs into MNs, the rosettes were lifted with dispase $(1 \mathrm{mg} / \mathrm{ml})$ and then cultured in suspension in NDM with $0.5 \mu \mathrm{M}$ RA, $0.1 \mu \mathrm{M}$ Pur, $10 \mathrm{ng} / \mathrm{ml}$ brain-derived neurotrophic factor (BDNF, PeproTech), $10 \mathrm{ng} / \mathrm{ml}$ glial cell line-derived neurotrophic factor (GDNF, PeproTech), and $10 \mathrm{ng} / \mathrm{ml}$ insulin-like growth factor (IGF, PeproTech) for 6 days. On day 19, HB9-positive MN spheres formed. To induce further maturation, these MN spheres were dissociated with accutase (Invitrogen) into single neurons and cultured adherently 
TABLE 1: Primers used for reverse transcription polymerase chain reaction (RT-PCR).

\begin{tabular}{lcc}
\hline Gene & Primer sequence $\left(5^{\prime}-3^{\prime}\right)$ & Annealing $\left({ }^{\circ} \mathrm{C}\right)$ \\
\hline Nestin & F AGAAACAGGGCCTACAGAGC; R GAGGGAAGTCTTGGAGCCAC & $65^{\circ} \mathrm{C}$ \\
SOX2 & F CCCCCGGCGGCAATAGCA; R TCGGCGCCGGGGAGATACAT & $60^{\circ} \mathrm{C}$ \\
Olig2 & F CCCTAAAGGTGCGGATGCTT; R CTGGATGCGATTTGAGGAGC & $65^{\circ} \mathrm{C}$ \\
Pax6 & F CGGAGTGAATCA GCTCGGTG; R CCGCTTATACTGGGCTATTTTGC & $60^{\circ} \mathrm{C}$ \\
HB9 & F AGCACCAGTTCAAGCTCAACA; R ACCAAATCTTCACCTGGGTCTC & $65^{\circ} \mathrm{C}$ \\
GAPDH & F ACCACAGTCCATGCCATCAC; R TCCACCACC CTGTTGCTGTA & $60^{\circ} \mathrm{C}$ \\
\hline
\end{tabular}

with NDM, including $0.5 \mu \mathrm{M}$ RA, $0.1 \mu \mathrm{M}$ Pur, $0.1 \mu \mathrm{M}$ Compound E (Cpd E, Calbiochem), and the abovementioned neurotrophic factors, for more than 7 days. Then, MNs differentiated into mature MNs.

2.3. Coculture of iPSC-Derived MNs and C2C12 Cells. C2C12 cells, a mouse myoblast cell line, were obtained from the China Center for Type Culture Collection and cultured in growth medium (DMEM/F12 supplemented with $10 \%$ fetal bovine serum (FBS)) until the cells reached $60-70 \%$ confluence. Then, the medium was changed to differentiation medium (DMEM/F12 with $2 \%$ horse serum (HS)), and myoblasts cultured in this medium for 4 days differentiated into skeletal muscle cells. On day 19, UC005-derived MN spheres were dissociated into single MNs, the MNs were added to each dish of muscle cells, and the medium was replaced with NDM, which was supplemented with RA, Pur, BDNF, GDNF, and IGF. Within 1-2 days, the MNs projected axons, and after more than 1 week in culture, the MNs formed NMJs with the muscle cells.

2.4. Immunofluorescence. The cultured cells were placed on $12 \mathrm{~mm}$ cover slips, fixed in $4 \%$ paraformaldehyde (PFA; Sigma) for $10 \mathrm{~min}$ at room temperature, washed three times with phosphate-buffered saline (PBS, Geno, China), and treated with a permeabilizing and blocking buffer (10\% donkey serum, $0.225 \%$ Triton X-100) for 1 hour at room temperature. Then, the cells were incubated with the following primary antibodies: OCT4 (1:200, Abcam), Nanog (1:250, Abcam), SSEA4 (1:250, Abcam), TRA-1-60 (1:300, Abcam), SOX1 (1:300, Boster), SOX2 (1:300, Boster), Nestin (1:300, Abcam), Olig2 (1:500, Millipore), Pax6 ( $1: 100$, DSHB), HB9 (1:50, DSHB), Islet1 (1:250, Abcam), ChAT (1:100, Millipore), and TuJ1 (1:250, Abcam). All antibodies were diluted in antibody dilution buffer $(2 \%$ donkey serum, $0.05 \%$ Triton X-100), and the cells were incubated with the antibodies overnight at $4^{\circ} \mathrm{C}$. After three washing steps, the cells were incubated for 1 hour at room temperature with secondary antibodies: Alexa Fluor (488 or 555) donkey anti-mouse, donkey anti-rabbit, and donkey anti-goat. For the detection of AChR, the cocultured cells were incubated with Alexa Fluor 555-conjugated $\alpha$-BTX $\left(1 \mu \mathrm{g} / \mathrm{ml}\right.$, Invitrogen) for 1 hour at $37^{\circ} \mathrm{C}$ before fixation. The cells were then washed 2 times in PBS and fixed with $4 \%$ PFA. All cell samples were observed using an Olympus fluorescence microscope.
2.5. RNA Isolation and RT-PCR. The mRNA expression levels of neuronal markers were analyzed using reverse transcriptase polymerase chain reaction (RT-PCR). TRIzol (Sigma), chloroform, isopropanol, and DNase I were used for total RNA extraction. Then, $0.8 \mu \mathrm{g}$ isolated RNA was reverse-transcribed to cDNA using the PrimeScript ${ }^{\mathrm{TM}}$ RT Master Mix (TAKARA, Kyoto, Japan). The PCRs contained $1.5 \mu \mathrm{l}$ cDNA, $10 \mu \mathrm{l}$ Ex Taq (TAKARA), $0.8 \mu \mathrm{l}$ primers (Table 1), and $7.7 \mu$ l nuclease-free $\mathrm{H}_{2} \mathrm{O}$. The PCR conditions consisted of $98^{\circ} \mathrm{C}$ for $2 \mathrm{~min}$, followed by 40 cycles of $98^{\circ} \mathrm{C}$ for $10 \mathrm{sec}, 65^{\circ} \mathrm{C}$ for $30 \mathrm{sec}$, and $72^{\circ} \mathrm{C}$ for $30 \mathrm{sec}$. For the PCRs, GAPDH was chosen as a housekeeping gene.

2.6. Statistical Analysis. GraphPad Prism 7 Software (GraphPad Software) was used for statistical analysis. The presented results are from three independent experiments. Statistical significance was determined with the $t$-test, and the results are presented as the means \pm standard errors of the mean. The $P$ values ${ }^{*} P<0.01,{ }^{* *} P<0.001$, and ${ }^{* * *} P<0.0001$ were considered significant.

\section{Results}

3.1. Characterization of iPSCs. Before initiation of the differentiation process, we first analyzed the pluripotent properties and purity of the iPSCs. Both urine-iPSCs and B-iPSCs exhibited uniform undifferentiated morphology, including a round shape, large nucleoli, scant cytoplasm, and organized colonies, similar to the features of embryonic stem cells (ESCs) (Figure 1) [8]. All iPSC lines mentioned above, including UC005, UE017, and B-iPSC, were confirmed by immunofluorescence staining of the pluripotency markers, including OCT4, Nanog, TRA-1-60, and SSEA4 (Figure 2) [9].

3.2. Induction of NEPs by Small Molecules. To demonstrate the potential of urine-derived iPSCs to differentiate into MNs, we induced both B-iPSCs and urine-iPSCs (UE017, UC005) into MNs (Figure 1). The specification of MNs is determined by the following steps: neutralization, caudalization, and ventralization [10]. The first step, neutralization, was activated by the combined inhibition of the bone morphogenetic protein (BMP) and transforming growth factor beta (TGF $\beta$ ) signaling pathways [11]. The GSK-3 $\beta$ inhibitor promotes neural progenitor proliferation by stimulating the canonical Wnt signaling pathway, which contributes to the maintenance of neural precursors [12]. Based on these 

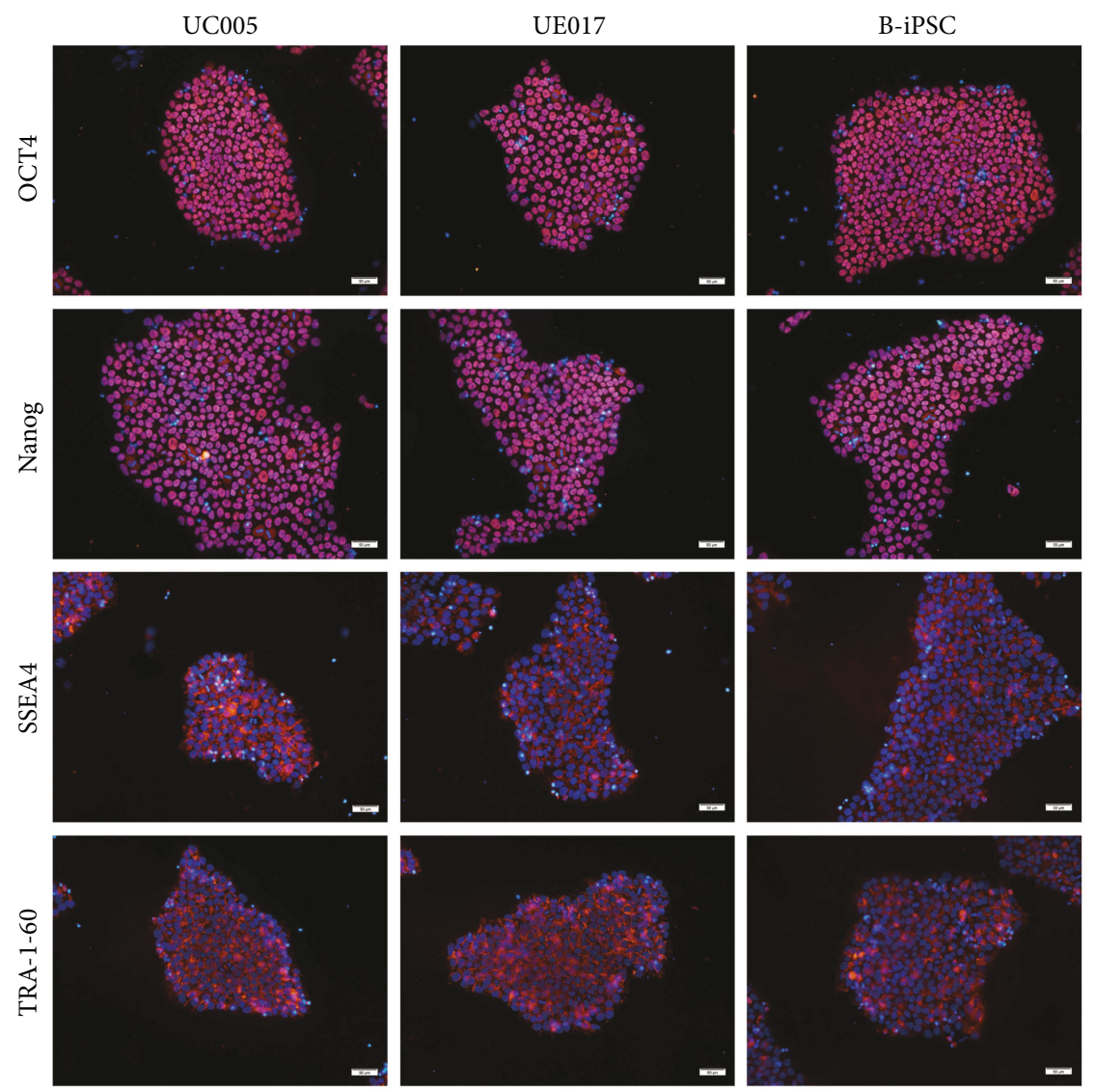

FIGURE 2: Pluripotency marker expression in iPSCs. Three iPSC lines, B-iPSC, UE017, and UC005, were positive for pluripotency markers, including OCT4, Nanog, SSEA4, and TRA-1-60. Cell nuclei were counterstained with 4',6-diamidino-2-phenylindole (DAPI). The scale bar is $50 \mu \mathrm{m}$.

preliminary studies $[7,13]$, when the confluence of the iPSC colonies reached 70-80\%, we dissociated the iPSCs, cultured them adherently in new Matrigel-coated plates, and then replaced the medium with NDM, which included DMH1 and SB431542 (inhibitors of BMP/TGF $\beta$ ) and CHIR99021 (inhibitor of GSK-3 $\beta$ ), to evoke neural induction. The iPSCs maintained under these neutralized conditions for 6 days differentiated into NEPs that exhibited obvious changes in morphology. The irregular, polygon-shaped cells aggregated centrally, and the peripheral cells were larger than the central cells (Figure 1(b)). Immunocytochemical staining revealed positive labeling for NEPs through the expression of neural progenitor markers SOX1, SOX2, and Nestin (Figure 3(a)) [14]. The majority of cells derived from all three iPSC lines expressed these pan-neural markers. By counting the positive cells, we determined that the positive ratio of NEPs derived from UC005 (UC005-NEP) with SOX1 and SOX2 immunolabeling was significantly higher than that of B-NEP and UE017-NEP (Figure 3(b)). The RT-PCR also confirmed the expression of the neural markers SOX2 and Nestin (Figure 4).

3.3. Efficient Neural Induction and MN Generation. The further induction of NEPs into MNPs refers to caudalization and ventralization, which are activated by the Sonic hedgehog (Shh) signaling pathway and RA [10]. The NEPs that were exposed to $0.1 \mu \mathrm{M}$ RA, $0.5 \mu \mathrm{M}$ Pur (an activator of the Shh signaling pathway), DMH1, SB, and CHIR for 6 days exhibited rapid changes in morphology. At this stage, the induced cells gathered centrally and formed rosettes (Figure 1(b)), neural tube-like structures with differentiation potential toward the central nervous system (CNS) and peripheral nervous system (PNS) fates [15]. The cells in the rosette were positive for the MNP markers [16], Olig2 and Pax6, but the peripheral cells bordering the rosette were negative (Figure 5(a)). Immunocytochemical staining and RT-PCR verified the expression of the MNP markers in both urine-iPSC- and B-iPSC-induced MNPs (Figures 4 and 5(a)). When we compared the ratios of cells positive for the MNP markers among the cells derived from all three iPSC lines, the expression levels of both Olig2 and Pax6 in cells derived from B-iPSCs (B-MNPs) and UC005 (UC005-MNPs) were significantly higher than those in cells from UE017 (UE017-MNPs); furthermore, the Pax6 expression levels in B-MNPs were significantly higher than those in UC005MNPs (Figure 5(c)).

3.4. MN Specification and Maturation. To induce MNPs into functional MNs, we cultured MNPs in suspension with decreased Pur $(0.1 \mu \mathrm{M})$ and increased RA $(0.5 \mu \mathrm{M})$ [7] coupled with BDNF, GDNF, and IGF (each $10 \mathrm{ng} / \mathrm{ml}$ ). After 6 days under the maturation conditions, $\mathrm{MN}$ floating spheres 

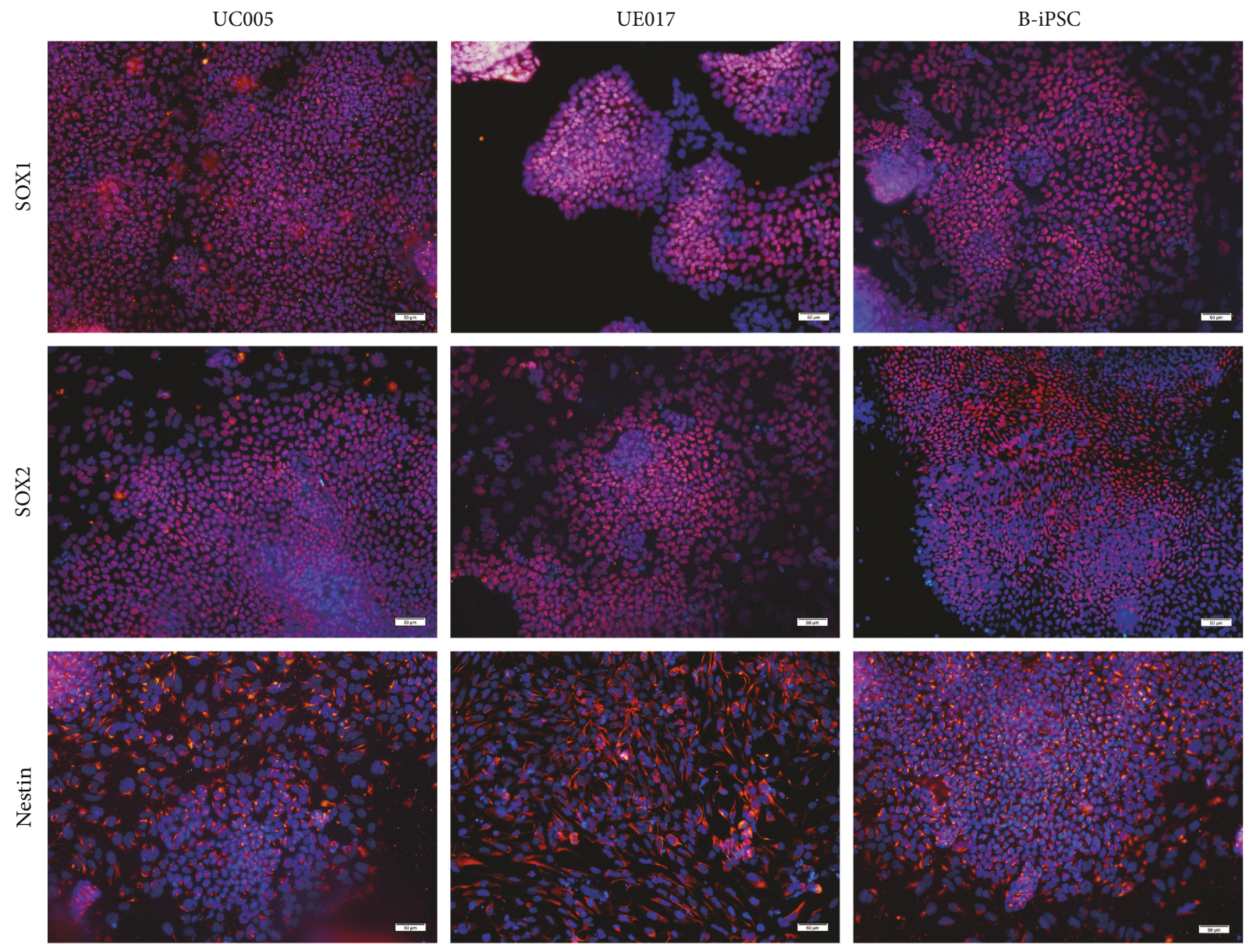

(a)

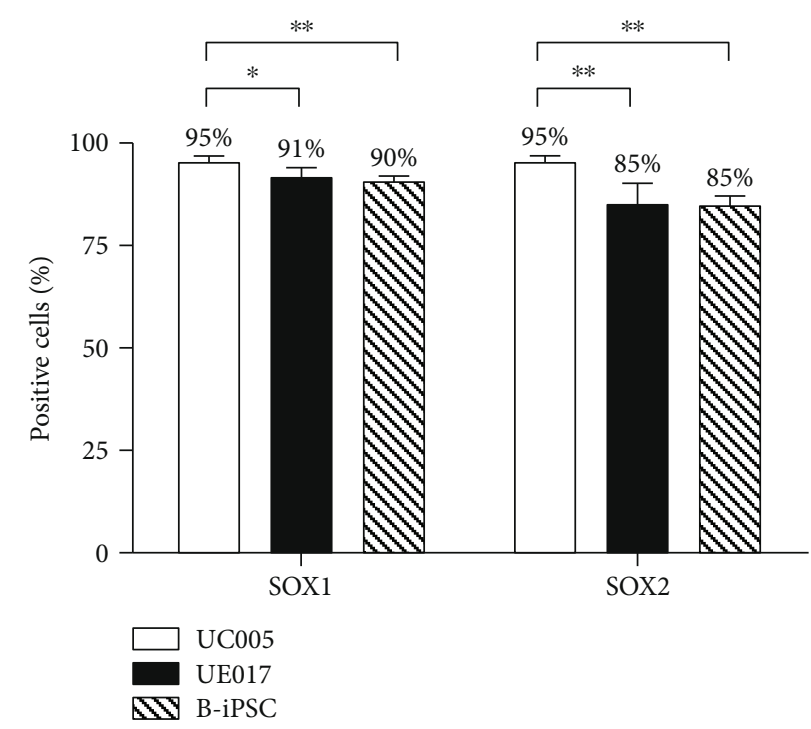

(b)

FIGURE 3: NEPs derived from urine-iPSCs and B-iPSCs express neural progenitor markers. (a) Immunocytochemical staining of neural progenitor markers. NEPs derived from all three iPSC lines, B-iPSC, UC005, and UE017, were positive for the neural precursor markers, SOX1, SOX2, and Nestin. Cell nuclei were counterstained with 4',6-diamidino-2-phenylindole (DAPI). The scale bar is $50 \mu \mathrm{m}$. (b) The ratio of positive labeling for SOX1 and SOX2 in NEPs after 7 days of induction. NEPs from UC005 expressed higher levels of neural precursor markers. The ratio of positive labeling $(\%)=$ (number of positive cells/total number $) \times 100 \%$. The results from three independent experiments are presented as the means \pm standard errors of the mean $(\mathrm{SEM}) .{ }^{* *} P<0.001$ and ${ }^{*} P<0.01$ according to Student's $t$-test. 


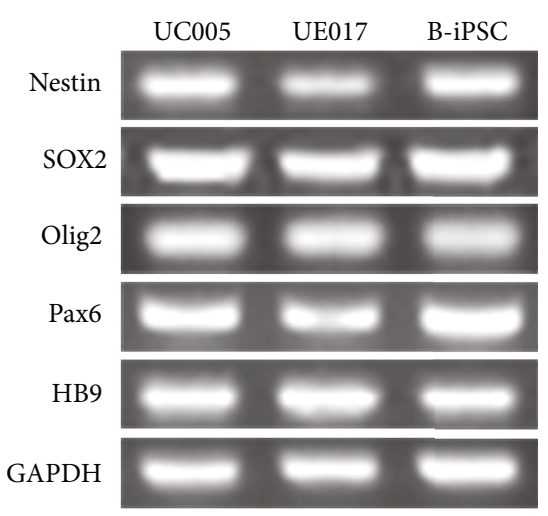

(a)

\begin{tabular}{cccc} 
NEP & MNP & MIN & Mature MN \\
I & I & । & । \\
\hline SOX1 & Olig2 & HB9 & ChAT \\
SOX2 & Pax6 & Islet1 & TuJ1 \\
Nestin & & &
\end{tabular}

(b)

Figure 4: (a) PCR analysis of the mRNA expression levels of neural cell markers at every differentiation stage. After 7 days of differentiation, both B-NEPs and urine-NEPs expressed the neural markers, Nestin and SOX2. After an additional 6 days of induction, cells derived from all three iPSC lines expressed the MNP markers, Olig2 and Pax6. As early as day 19 of induction, MNs from B-iPSCs and urine-iPSCs expressed the MN-specific marker, HB9. These mRNA expression results were consistent with the results of protein expression, as shown by immunofluorescence. (b) Stages of differentiation of MNs from iPSCs and markers commonly used for their characterization.

formed, as evidenced by the expression of HB9, an immature motor neuron marker [17], and LIM-homeodomain transcription factor Islet1 [18] (Figure 5(b)). The ratio of positive labeling for HB9 in B-iPSC-derived MNs (B-MNs) was higher than that in MNs derived from UC005 (UC005MNs) and UE017 (UE017-MNs), but the difference was not statistically significant (Figure 5(d)). We tried to culture cells adherently in this stage to avoid damaging the dissociated spheres in the next stage; however, the ratio of positive labeling decreased. Then, we dissociated these MN spheres into single cells and cultured them on Matrigel with Pur, RA, and Cpd E (an inhibitor of NOTCH). The next day, the adherent urine-MNs and B-MNs projected axons. When the MNs were cultured under these conditions for more than 7 days, the MNs matured even further and projected long axons; axonal elongation occurred gradually over time (Figure 1(b)). MN maturation was evidenced by immunofluorescence staining of the neural marker TuJ1 and the mature MN-specific marker ChAT (Figure 6(a)) [19, 20]. RT-PCR further verified the mRNA expression of the MN-specific marker HB9 in both urine-MNs and B-MNs, consistent with immunofluorescence observations (Figure 4).

3.5. NMJs Formed between Urine-MNs and Muscle Cells. On day 19 of neural differentiation, dissociated UC005MNs were added to muscle culture cell dishes, and after 5 days of coculturing, muscle cell contraction was observed with a brightfield microscope. Within additional 2-3 days, immunofluorescence staining revealed positive labeling for the acetylcholine receptor (AChR) marker, $\alpha$-bungarotoxin ( $\alpha$-BTX), on the surface of the muscle cells [21] and colocalization of $\alpha$-BTX and TuJ1 at sites on the axons of the UC005-MNs (Figure 6(b)), which indicated accumulation of AChR and the formation of NMJs between urine-MNs and muscle cells at these sites [22].

\section{Discussion}

Directed differentiation of stem cells into MNs holds a great promise for the in vitro modeling of neurodegenerative diseases and cellular replacement therapies [23]. Several protocols for MN differentiation have been reported [7, 24-26]. Among these protocols, Du's protocol yielded a highly pure population of MNs that differentiated from iPSCs. To date, different sources of iPSCs, including skin fibroblasts and blood cells, have been used in these $\mathrm{MN}$ differentiation protocols $[27,28]$. Compared to skin fibroblasts and blood cells, urinary cells are safer and more efficient for isolation and reprogramming [4]. In this study, for the first time, we demonstrate the potential of urine-iPSCs to differentiate into MNs. We further investigated the capacities of blood cellderived iPSCs and urine-iPSCs to differentiate into MNs by comparing the expression levels of neural markers.

We used Du's differentiation protocol [7], with slight modification, to induce MNPs to differentiate into mature MNs. To promote steady differentiation, the cells were treated with RA, Pur, Cpd E, and neurotrophic factors, which were not consistently added in Du's protocol. Before the differentiation of iPSCs into MNs, urine-iPSCs (UC005, UE017) exhibited characteristic properties of PSCs, which was confirmed by immunofluorescence staining of pluripotency markers. Then, activation induced by BMP and TGF $\beta$ combined with inhibition of GSK-3 $\beta$ initiated the neutralization of iPSCs. After 7 days of induction, both B-iPSCs and urine-iPSCs differentiated into NEPs. The ratios of positive labeling for the neural precursor markers in NEPs derived from the three iPSC lines were all greater than $85 \%$, and the ratio of the UC005-NEPs was slightly higher than those of the other two cell lines. During the subsequent 6 days, the presence of RA and Pur resulted in rapid changes in morphology, formation of rosettes, and upregulation of the MNP markers Olig2 and Pax6. The expression levels of the MNP markers in the UC005-MNPs and B-MNPs were significantly higher than those in the UE017-MNPs. With further differentiation in suspension, cells derived from all three iPSC lines congregated and formed MN spheres, which expressed the MN markers HB9 and Islet1. The rates of positive labeling for the precursor neural markers and MNP markers were significantly different among the cells from UC005, UE017, and B-iPSC, but the rates of positive labeling for the MN marker, HB9, were not statistically significant among the cells. For the further MN maturation, the combined actions of RA, Pur, and Cpd E resulted in the projection of long axons and expression of the mature $\mathrm{MN}$ marker, ChAT, in MNs derived from all three iPSC lines. When cocultured with muscle cells, urine-iPSC-derived MNs 

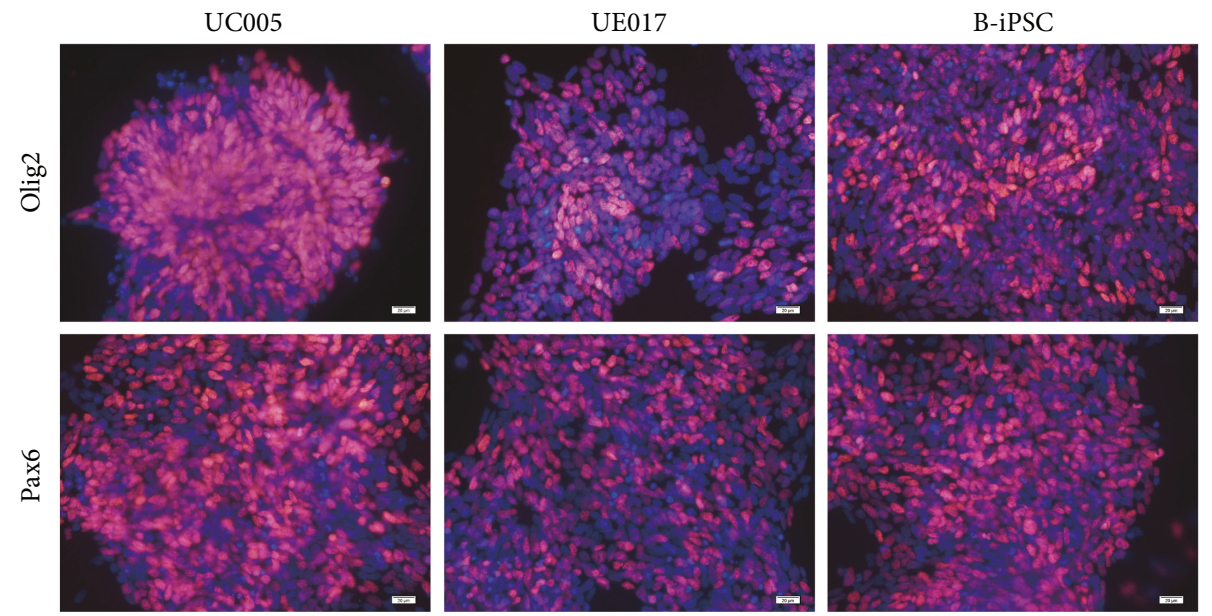

(a)
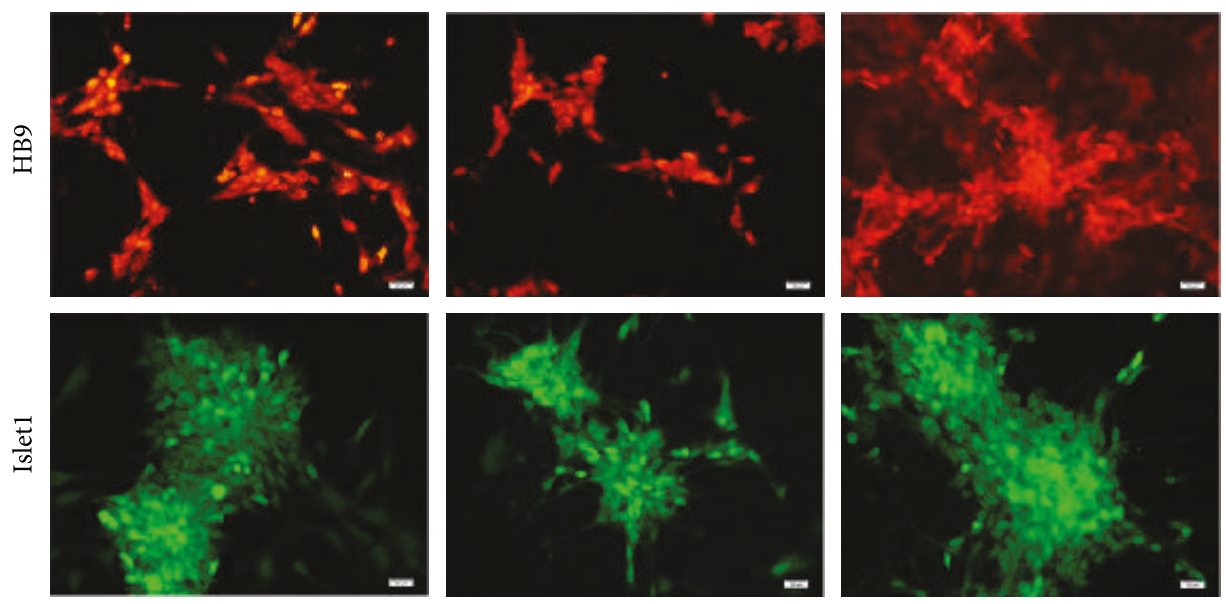

(b)

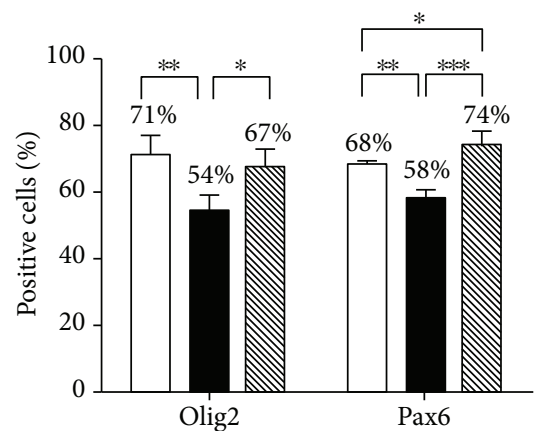

$\square$ UC005

- UE017

B-iPSC

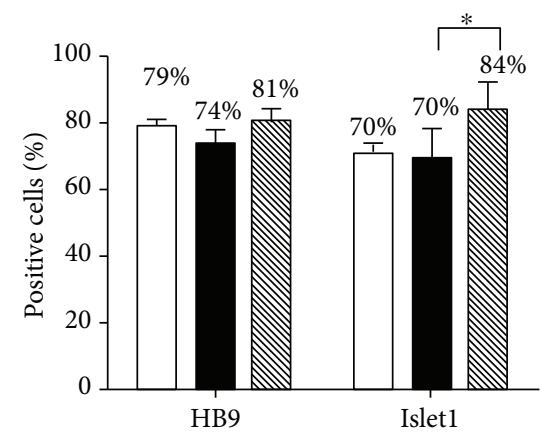

$\square$ UC005

- UE017

B-iPSC

(c)

(d)

FIGURE 5: Immunocytochemical staining and ratios of positive labeling of the MNPs and MNs. (a) After 13 days of induction, cells derived from all three iPSC lines, UC005, UE017, and B-iPSC, were positive for the MNP markers, Olig2 and Pax6. Cell nuclei were counterstained with 4',6-diamidino-2-phenylindole (DAPI). The scale bar is $50 \mu \mathrm{m}$. (b) After 19 days of induction, the cells derived from all three iPSC lines, UC005, UE017, and B-iPSC, were positive for the MN markers, HB9 and Islet1. Cell nuclei were counterstained with 4',6-diamidino-2phenylindole (DAPI). The scale bar is $50 \mu \mathrm{m}$. (c) Cells from the UC005 and B-iPSC lines expressed higher levels of the MNP markers than the cells from the UE017 line. (d) The ratios of positive labeling for HB9 and Islet1 in MNPs after 19 days of induction. The ratios of positive labeling for HB9 were 79\%, 74\%, and 81\% in MNs derived from UC005, UE017, and B-iPSC, respectively, but the difference was not statistically significant. A total of $84 \%$ of MNs from B-iPSC expressed Islet1, which was significantly higher than that of MNs from UC005 and UE017. The ratio of positive labeling $(\%)=$ (number of positive cells/total number) $\times 100 \% .{ }^{* * *} P<0.0001,{ }^{* *} P<0.001$, and ${ }^{*} P<0.01$ according to Student's $t$-test. 

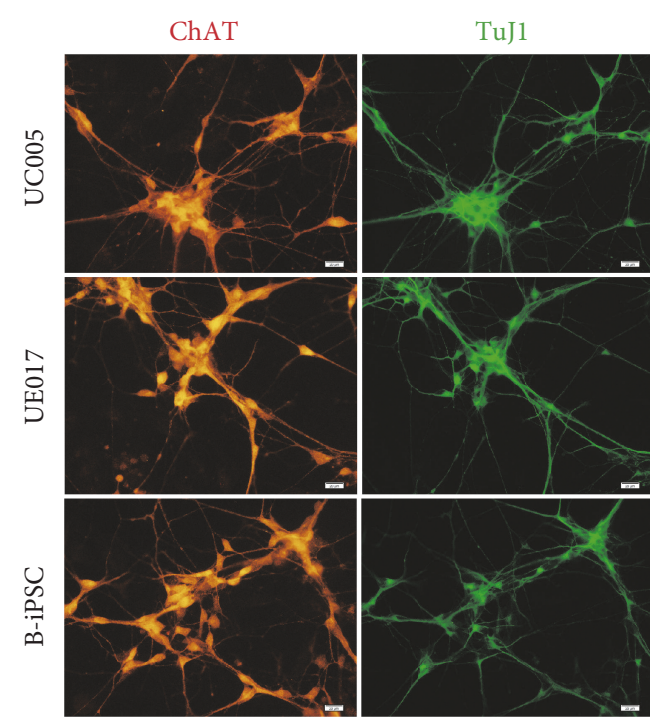
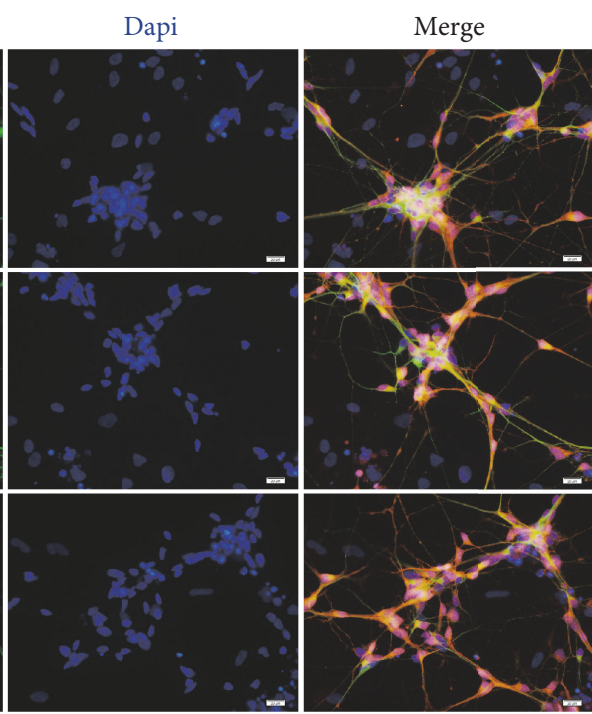

(a)
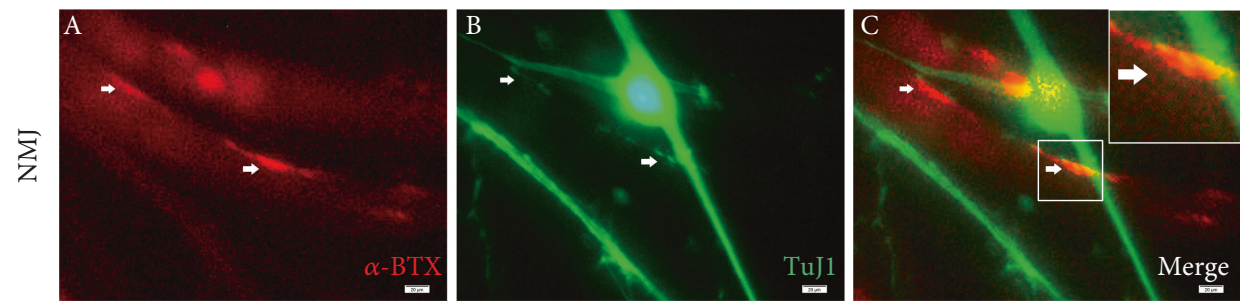

(b)

Figure 6: Mature and functional MNs were generated after 26 days of differentiation. (a) Immunocytochemical staining of mature MNs. Under inductive conditions for more than 26 days, cells derived from all three iPSC lines, B-iPS, UE017, and UC005, expressed the mature MN marker choline acetyltransferase (ChAT). They also expressed the neuronal marker TuJ1. Cell nuclei were counterstained with 4',6-diamidino-2-phenylindole (DAPI). (b) Cocultured muscle cells and MNs form neuromuscular junctions. Immunofluorescence staining shows $\alpha$-bungarotoxin- (BTX-) positive sites overlaid with TuJ1 labeling in the UC005 MNs (A, B, C). Cell nuclei were counterstained with $4^{\prime}, 6$-diamidino-2-phenylindole (DAPI). The scale bar is $50 \mu \mathrm{m}$.

exhibited functional properties, including the projection of axons toward muscle cells, induction of muscle contractions, and NMJ formation. To further confirm our immunocytochemical observations, we used RT-PCR to analyze the mRNA expression levels of neuronal- and MN-specific markers. In all three iPSC lines, we detected expression of neuronal markers (SOX1, Nestin, Pax6, and Olig2) and the MN-specific marker, HB9.

In conclusion, the expression profiles of neural cell markers, as measured by immunocytochemistry and RTPCR, demonstrated that urine-iPSCs were able to successfully differentiate into MNs. The formation of NMJs between MNs and muscle cells further indicated the functional properties of urine-MNs. In addition, the comparison of the ratios of positive labeling for MN markers demonstrated that the capacity of urine-iPSCs and B-iPSCs to differentiate into MNs is not significantly different. However, there are some important aspects that we did not investigate, such as the electrophysiological properties of induced MNs in vitro [29]. Furthermore, transplantation of MNs into an MND animal model is necessary for future studies of cell regenerative medicine [30]. These two aspects mentioned above will be explored in our future research. In this study, we demonstrated the feasibility of differentiating urine-derived iPSCs into MNs. Since urinederived iPSCs can be easily accessed in a noninvasive manner, urine-derived iPSCs provide a novel platform for disease modeling, drug screening, and cellular therapy for MND.

\section{Conflicts of Interest}

The authors declare that they have no conflicts of interest.

\section{Authors' Contributions}

Huan Yi carried out the experiments, analyzed the data, and wrote the draft. Bingbing Xie, Ben Liu, Xuan Wang, Li Xu, Jia Liu, and Min Li collected and analyzed the data. Xiufeng Zhong and Fuhua Peng conceived and designed the experiments and wrote and approved the manuscript. Fuhua Peng supervised the project.

\section{Acknowledgments}

The authors would like to thank Professor Guangjin Pan (Chinese Academy of Sciences, Guangzhou Institutes of Biomedicine and Health) for providing two urine 
cell-derived iPSC lines, UE017 and UC005. This study was supported by the Natural Science Foundation of Guangdong Province (no. 2015A03013167), the Guangzhou Science and Technology Project Fund (1563000227), the National Natural Science Foundation of China (81271327 and 81570874), the Science and Technology Project of Guangdong Province (2014A020211008, 2015A020212011, and 2017B020230003), and National Key Research and Development Program of China (2016YFC1101103, 2017YFA0104101, and 2012CB966500).

\section{References}

[1] I. Faravelli, M. Bucchia, P. Rinchetti et al., "Motor neuron derivation from human embryonic and induced pluripotent stem cells: experimental approaches and clinical perspectives," Stem Cell Research \& Therapy, vol. 5, no. 4, p. 87, 2014.

[2] J. S. Lunn, S. A. Sakowski, T. Federici, J. D. Glass, N. M. Boulis, and E. L. Feldman, "Stem cell technology for the study and treatment of motor neuron diseases," Regenerative Medicine, vol. 6, no. 2, pp. 201-213, 2011.

[3] Y. Shi, H. Inoue, J. C. Wu, and S. Yamanaka, "Induced pluripotent stem cell technology: a decade of progress," Nature Reviews Drug Discovery, vol. 16, no. 2, pp. 115-130, 2017.

[4] C. Wang, F. Hei, Z. Ju, J. Yu, S. Yang, and M. Chen, "Differentiation of urine-derived human induced pluripotent stem cells to alveolar type II epithelial cells," Cellular Reprogramming, vol. 18, no. 1, pp. 30-36, 2015.

[5] S. Z. Zhang, H. F. Li, L. X. Ma, W. J. Qian, Z. F. Wang, and Z. Y. Wu, "Urine-derived induced pluripotent stem cells as a modeling tool for paroxysmal kinesigenic dyskinesia," Biology Open, vol. 4, no. 12, pp. 1744-1752, 2015.

[6] Y. Xue, X. Cai, L. Wang et al., "Generating a non-integrating human induced pluripotent stem cell bank from urinederived cells," PLoS One, vol. 8, no. 8, article e70573, 2013.

[7] Z. Du, H. Chen, H. Liu et al., "Generation and expansion of highly pure motor neuron progenitors from human pluripotent stem cells," Nature Communications, vol. 6, p. 6626, 2015.

[8] K. Takahashi and S. Yamanaka, "Induction of pluripotent stem cells from mouse embryonic and adult fibroblast cultures by defined factors," Cell, vol. 126, no. 4, pp. 663-676, 2006.

[9] K. Takahashi, K. Tanabe, M. Ohnuki et al., "Induction of pluripotent stem cells from adult human fibroblasts by defined factors," Cell, vol. 131, no. 5, pp. 861-872, 2007.

[10] H. Wichterle, I. Lieberam, J. A. Porter, and T. M. Jessell, "Directed differentiation of embryonic stem cells into motor neurons," Cell, vol. 110, no. 3, pp. 385-397, 2002.

[11] B. Bragdon, O. Moseychuk, S. Saldanha, D. King, J. Julian, and A. Nohe, "Bone morphogenetic proteins: a critical review," Cellular Signalling, vol. 23, no. 4, pp. 609-620, 2011.

[12] E. Hur and F. Zhou, "GSK3 signalling in neural development," Nature Reviews Neuroscience, vol. 11, pp. 539-551, 2010.

[13] W. Liu and G. Chen, "Cryopreservation of human pluripotent stem cells in defined medium," Current Protocols in Stem Cell Biology, vol. 31, pp. 1C.17.1-1C.17.13, 2014.

[14] V. Graham, J. Khudyakov, P. Ellis, and L. Pevny, "SOX2 functions to maintain neural progenitor identity," Neuron, vol. 39, no. 5, pp. 749-765, 2003.

[15] Y. Elkabetz, G. Panagiotakos, G. Al Shamy, N. D. Socci, V. Tabar, and L. Studer, "Human ES cell-derived neural rosettes reveal a functionally distinct early neural stem cell stage," Genes \& Development, vol. 22, no. 2, pp. 152-165, 2008.

[16] Q. R. Lu, T. Sun, Z. Zhu et al., "Common developmental requirement for Olig function indicates a motor neuron/oligodendrocyte connection," Cell, vol. 109, no. 1, pp. 75-86, 2002.

[17] J. T. Dimos, K. T. Rodolfa, K. K. Niakan et al., "Induced pluripotent stem cells generated from patients with ALS can be differentiated into motor neurons," Science, vol. 321, no. 5893, pp. 1218-1221, 2008.

[18] H. F. Wang and F. C. Liu, "Developmental restriction of the LIM homeodomain transcription factor Islet-1 expression to cholinergic neurons in the rat striatum," Neuroscience, vol. 103, no. 4, pp. 999-1016, 2001.

[19] M. Naujock, N. Stanslowsky, P. Reinhardt et al., "Molecular and functional analyses of motor neurons generated from human cord-blood-derived induced pluripotent stem cells," Stem Cells and Development, vol. 23, no. 24, pp. 3011-3020, 2014.

[20] S. L. Rossi, G. Nistor, T. Wyatt et al., "Histological and functional benefit following transplantation of motor neuron progenitors to the injured rat spinal cord," PLoS One, vol. 5, no. 7, article e11852, 2010.

[21] E. Bruneau, D. Sutter, R. I. Hume, and M. Akaaboune, "Identification of nicotinic acetylcholine receptor recycling and its role in maintaining receptor density at the neuromuscular junction in vivo," The Journal of Neuroscience, vol. 25, no. 43, pp. 9949-9959, 2005.

[22] J. A. Umbach, K. L. Adams, C. B. Gundersen, and B. G. Novitch, "Functional neuromuscular junctions formed by embryonic stem cell-derived motor neurons," PLoS One, vol. 7, no. 5, article e36049, 2012.

[23] E. Kiskinis and K. Eggan, "Progress toward the clinical application of patient-specific pluripotent stem cells," Journal of Clinical Investigation, vol. 120, no. 1, pp. 51-59, 2010.

[24] B. Hu and S. Zhang, "Differentiation of spinal motor neurons from pluripotent human stem cells," Nature Protocols, vol. 4, no. 9, pp. 1295-1304, 2009.

[25] J. S. Toma, B. C. Shettar, P. H. Chipman et al., "Motoneurons derived from induced pluripotent stem cells develop mature phenotypes typical of endogenous spinal motoneurons," Journal of Neuroscience, vol. 35, no. 3, pp. 1291-1306, 2015.

[26] M. W. Amoroso, G. F. Croft, D. J. Williams et al., “Accelerated high-yield generation of limb-innervating motor neurons from human stem cells," Journal of Neuroscience, vol. 33, no. 2, pp. 574-586, 2013.

[27] E. Kiskinis, J. Sandoe, L. A. Williams et al., "Pathways disrupted in human ALS motor neurons identified through genetic correction of mutant SOD1," Cell Stem Cell, vol. 14, no. 6, pp. 781-795, 2014.

[28] S. M. Wu and K. Hochedlinger, "Harnessing the potential of induced pluripotent stem cells for regenerative medicine," Nature Cell Biology, vol. 13, no. 5, pp. 497-505, 2011.

[29] K. A. Puttonen, M. Ruponen, N. Naumenko, O. H. Hovatta, P. Tavi, and J. Koistinaho, "Generation of functional neuromuscular junctions from human pluripotent stem cell lines," Frontiers in Cellular Neuroscience, vol. 9, 2015.

[30] L. Xu, J. Yan, D. Chen et al., "Human neural stem cell grafts ameliorate motor neuron disease in SOD-1 transgenic rats," Transplantation, vol. 82, no. 7, pp. 865-875, 2006. 


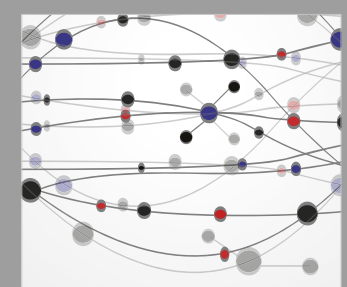

The Scientific World Journal
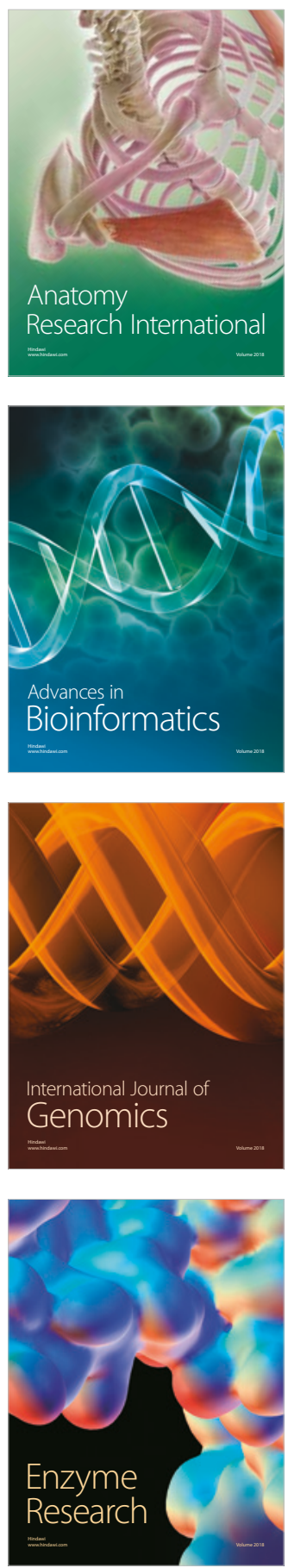
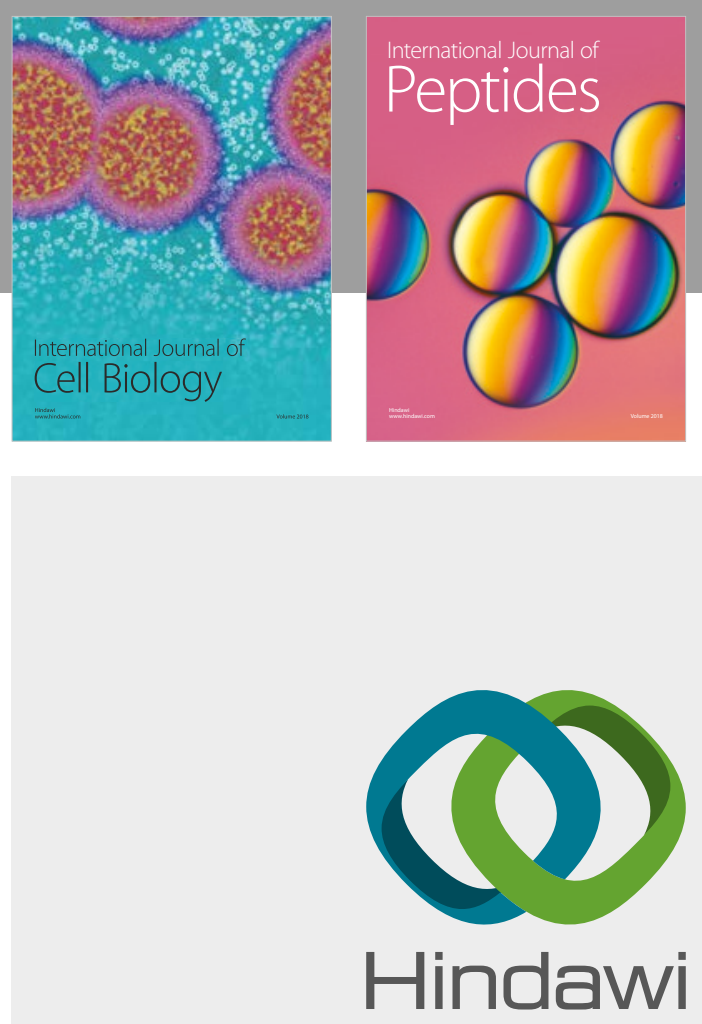

Submit your manuscripts at

www.hindawi.com
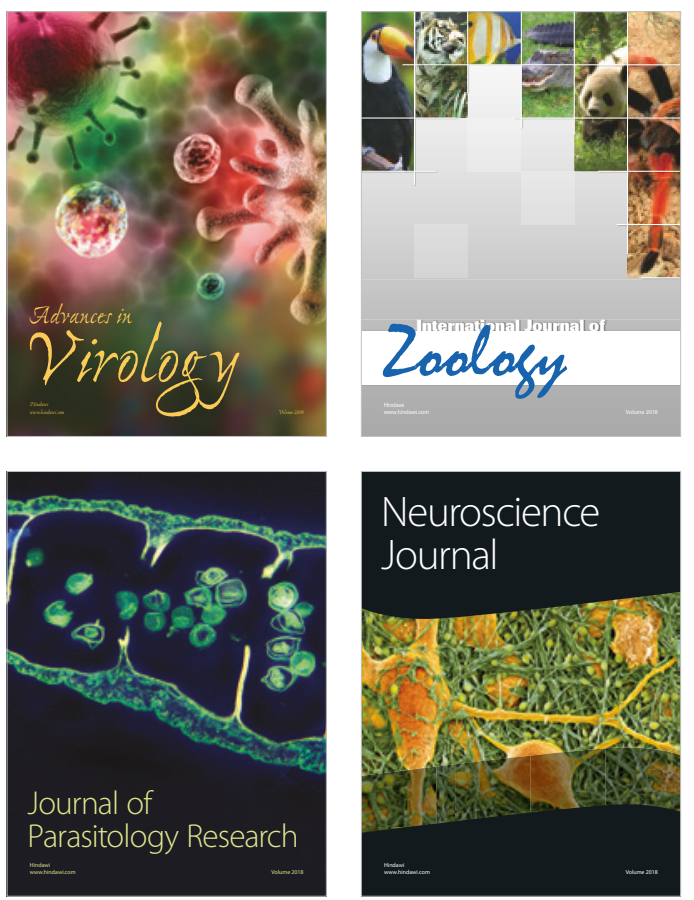
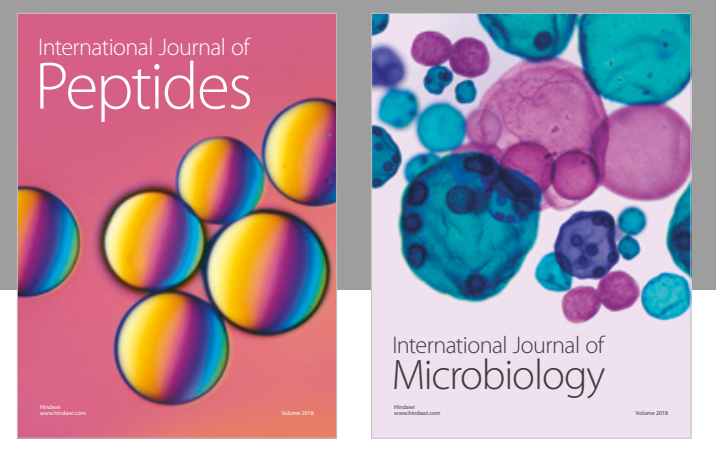

nternational Journal of Microbiology
Journal of
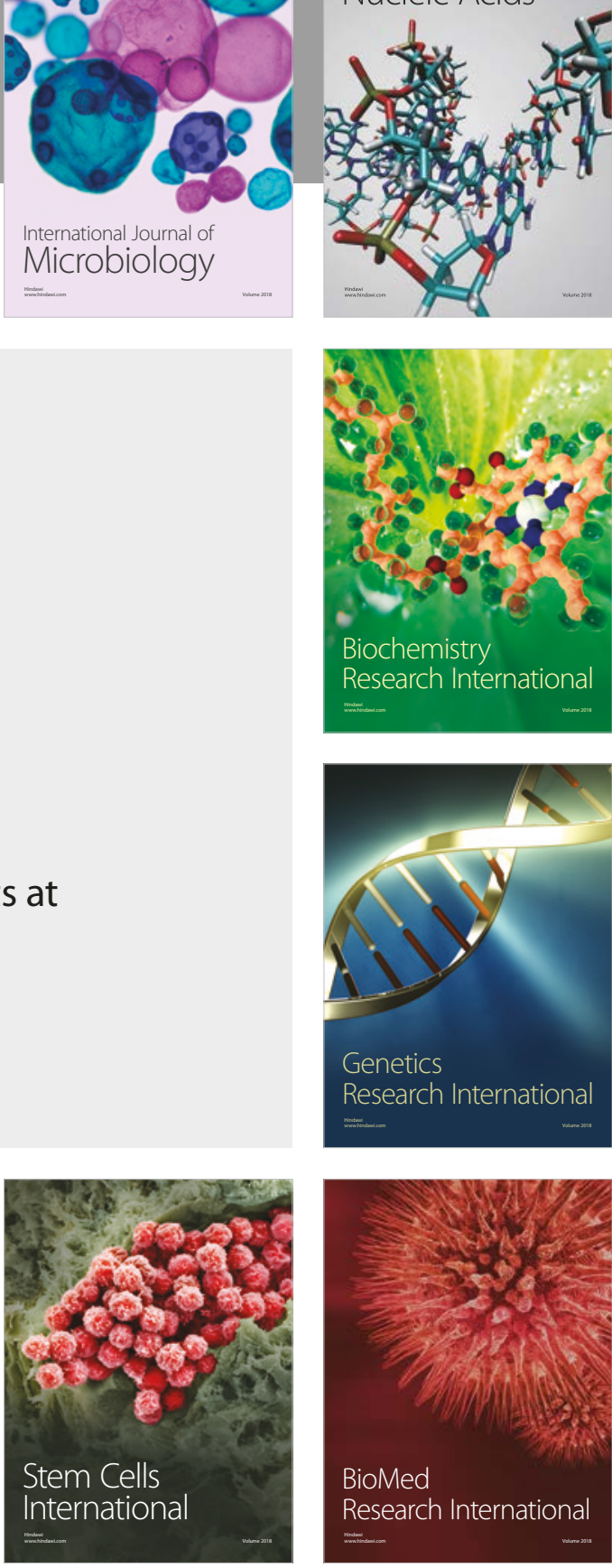
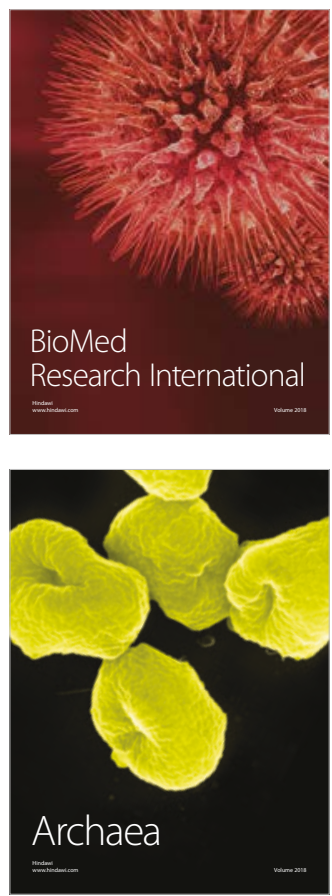